\title{
Response to "The Value of Angio CT Multislice for Pre-operative Assessment and Prediction of Post PCI Successful Intervention in Acute Coronary Syndromes Associated by Left Main Disease"
}

\author{
Monica Marton-Popovici \\ Swedish Medical Center, Department of Internal Medicine and Critical Care, Edmonds, Washington, USA
}

To the editor

I read with great interest the article entitled "The Value of Angio CT Multislice for Pre-operative Assessment and Prediction of Post PCI Successful Intervention in Acute Coronary Syndromes Associated by Left Main Disease", published by Blendea et al in issue 1/2015 of JCE journal [1]. As the authors pointed out, Angio CT assessment of culprit lesions located in the left main can provide complex information regarding the severity of the disease and could be very useful for evaluation of the risk of post-procedural complications. At the same time, the study demonstrates that use of Angio CT can increase the indication for PCI in complex left main lesions.

However, it should be noted that the study included only patients with acute coronary syndromes (ACS), fact with two consequences. First, it is well-known that ACS patients can present Angio CT characteristics that are significantly different from the general population with coronary artery disease [2]. An ACS is usually resulting from the rupture of a vulnerable coronary plaque and these unstable coronary plaques exhibit particular CT characteristics such as a large low-density core, spotty calcifications or vascular remodeling. These characteristics are not included in the calculation of Angio CT Syntax score, however they are particularly important for prediction of procedure-related risk.
Secondly, ACS is a general frame that includes ST-elevation acute myocardial infarction (STEMI), non ST-segment elevation acute myocardial infarction (NSTEMI) and unstable angina. While the Angio CT assessment could be accepted as an indication for pre-operative assessment in the last two categories (NSTEMI and unstable angina), in STEMI cases the percutaneous revascularization should be performed as fast as possible, without any delay, as indicated by the current guidelines [3]. Therefore any procedure that could lead to any unnecessary prolongation of the time from the onset of symptoms to revascularization should be avoided. The distinction between the three different types of ACS resulting from rupture of unstable plaques located in the left main is not clearly presented in this study, while the therapeutic strategy is significantly different for STEMI cases than for NSTEMI or unstable angina cases. This is particularly important, in my opinion, as the study refers to the value of Angio CT for pre-operative assessment of coronary lesions and for assessment of further post-operative risk, both aspects being significantly different in the STEMI population than in NSTEMI or unstable angina patients.

An extension of this study, in order to reveal the potential differences between these two categories, together with a more detailed description of Angio CT aspects characteristic for vulnerable plaques in culprit lesions located in the left main, could add significant further value to this interesting study. 


\section{REFERENCES}

1. Blendea C, Kovacs I, Barcan A, Nyulas T, Chitu M. The value of Angio CT Multislice for Pre-operative Assessment and Prediction of Post PCI Successful Intervention in Acute Coronary Syndromes Associated by Left Main Disease. J Cardiovasc Emmerg. 2015;1:2-34.
2. Motoyama S, Ito H, Sarai M, et al. Plaque Characterization by Coronary Computed Tomography Angiography and the Likelihood of Acute Coronary Events in Mid-Term Follow-Up. J Am Coll Cardiol. 2015;66:337-346.

3. Steg G, James SK, Atar D, etal. ESC Guidelines for themanagement of acute myocardial infarction in patients presenting with STsegment elevation. Eur Heart J. 2012; 33:2569-2626. 